\title{
The Problem of Prediction in Artificial Intelligence and Synthetic Biology
}

\author{
Francesco Bianchini \\ Department of Philosophy and Communication Studies \\ University of Bologna \\ Via Zamboni 38, 40126 Bologna, Italy
}

\begin{abstract}
The problem of prediction is a general problem in the philosophy of science. It is important in every discipline for which prediction concerns the behavior of an artificial or a biological system, such as artificial intelligence or synthetic biology. Synthetic biology shares with artificial intelligence some theoretical issues from the point of view of prediction. My claim is that the problems related to the prediction of system behaviors are analogous because: $(a)$ artificial intelligence and synthetic biology aim at producing autonomous systems; and $(b)$ their products interact with an open-ended and uncertain context. I argue my claim by providing three versions of the prediction problem in artificial intelligence and synthetic biology, to show the analogies between them within this framework and to suggest some useful consequences.
\end{abstract}

Keywords: artificial intelligence; synthetic biology; prediction; autonomous systems; philosophy of science

\section{Introduction}

The problem of prediction is a general problem in the philosophy of science. Prediction in science is related to the scientific method and consists of formulating hypotheses as the logical consequences of scientific theories and testing them through observations or experiments. A theory is stronger if its predictive power is corroborated by experience. In some disciplines, prediction has a slightly different meaning and relevance. In artificial intelligence (AI) and synthetic biology (SB), prediction is related to actual products, which are artificial artifacts or artificial biological entities, whose behavior has to be tested as well as controlled for specific goals. The former case is more typical of the AI methodology of modeling "intelligent" capabilities; the latter is more typical of SB products and their usefulness for practical purposes (medical or biotechnological applications, for example). However, both are interested in predicting the behavior of their actual outcomes, that is, the entities they aim at building. This is the specific sense of prediction that I will address in what follows, showing how this particular issue connects $\mathrm{AI}$ and SB in some respects. 
SB shares with AI and computer science some theoretical issues, which are influential from the point of view of applied research. For example, present-day research on genome editing reuses old parallelisms between the genetic code and the theory of information. Genome editing is seen as a sort of programming, and programming is par excellence the science of information. My claim is that the problems of $\mathrm{AI}$ and $\mathrm{SB}$ are related to the prediction of a system's behaviors that are analogous because of the following: (1) AI and SB aim at producing autonomous systems; and (2) AI and SB products interact with an open-ended and uncertain context, such as real-world environments. The notion of autonomy is currently debated, especially in AI, from both an epistemological and an ethical, legal and political point of view. Generally speaking, an autonomous system, software or robotics, is a system that goes beyond the behavior programmed in the initial algorithm. In particular, the autonomous system can learn by itself from data and environment, so that its behavior is unpredictable. It usually performs in a context, that is, a social, virtual or actual environment, in interaction with other natural or artificial entities. The contextual behavior is unpredictable because it depends on an indefinite number of variables that can influence the behavior itself (see, for example, [1]). In a similar sense, an SB entity or a biological entity modified with SB techniques (see, for example, [2]) acts in a context at different levels, from cellular to real world, in interaction with all the components of its context. The "programmed" nature of these entities, along with their complexity, or the complexity of the contextual system, makes it hard to predict the results and the behavior of the system in advance. These kinds of SB entities are autonomous in an analog sense with AI autonomous systems, even though they are generated in a different manner.

This is just a part of what I call the "prediction problem" in AI and SB. Fresh problems encountered from this standpoint are analogous to problems in AI emergent systems, such as those produced by means of cellular automata and evolutionary programming. The notion of emergence has been debated in the philosophy of science since the 1960s [3]. A typical distinction is between weak emergence and strong emergence [4]. While the former regards the arising of new properties or entities as a result of the interactions of entities at a lower level and is connected to the assumptions that make it possible to simulate a system-at an elementary level—that is emergent or shows emergent properties, the latter emphasizes the irreducibility of high-level properties or entities to the lower ones [5]. The debate is still open, and the relevance of this notion is clear for the biological world as well as for artificial systems that simulate biological phenomena or are bio-inspired, as in many recent trends for AI and robotics. In $\mathrm{SB}$ and $\mathrm{AI}$, the epistemological debate on emergence is related to 
the biological complexity of natural and synthetic systems and the practical limitations on prediction and control of living systems [6, 7]. The connection between emergence and predictability is therefore strong from a computational and an epistemological point of view.

There is another theoretical issue related to the general aim of these two disciplines. In a technological improved scenario, genetic manipulation, selection and engineering could lead to biological superintelligence, and evolutionary computation is a good candidate to achieve an artificial superintelligence. The question about how evolutionary computation could achieve artificial superintelligence concerns the overall problem of prediction in AI and the relationship with SB, which can provide autonomous systems and systems interacting in an unpredictable context.

In what follows, I will clarify and support my claim about prediction in AI and SB and the relationship between them. In Section 2, I speak about the general notion of prediction; in Section 3, I deal especially with the problem of prediction in $\mathrm{AI}$ and SB; in Section 4, I provide three versions of the problem; and Section 5 has the concluding remarks.

\section{The Notion of Prediction and the Problem of Prediction}

Prediction is to foretell what will happen on the basis of what is known. In science, prediction provides a specific future situation that will take place given some initial conditions and the scientific laws and theories concerning the specific scientific domain in question. More specifically, prediction in science has an important role in research and discovery, insofar as it allows for testing scientific theories under various circumstances. Prediction is important in many fields related to science: probability, statistics, game theory, decision theory, causality, scientific theory, scientific models, models in science, future technological developments and simulations. For the aims of this paper, future technological developments and simulations are the most prominent.

In general philosophy of science, the issue of prediction is usually connected with the problem of scientific explanation. There is a longlasting debate at least since Hempel-Oppenheim's proposal for the deductive-nomological model of explanation of 1948 [8]. This model means to be adequate for explanations and predictions of phenomena, which are seen as a relation of symmetry. Many authors have questioned the validity of the symmetry assumption with different arguments, especially concerning the causal explanation $[9,10]$. The difference between explanation and prediction concerns the fact that the former refers to something that has already happened and the 
latter to something that will take place, or what should take place. Therefore, we may consider explanation and prediction as opposing in the light of their temporal asymmetry.

We may also consider prediction as part of the pragmatic aspect of science, as something that science is used for, especially to control nature, such as in applied sciences. Without prediction, there is no control of scientific results and artifacts. This goal is, however, uncertain, because the more complex the system, the less accurate the prediction. Prediction shows, therefore, that science and scientific knowledge have limits [11, 12]. Prediction is limited compared to explanation and is subject to uncertainty and to every cognitive issue related to uncertainty: bounded rationality, cognitive inaccessibility and the relevance problem. The reduction of uncertainty strengthens the power of prediction, but uncertainty is unavoidable and ineliminable, especially with the increasing complexity of systems, as in the case of living systems or systems simulating living ones. This is precisely the domain I deal with, that is, prediction in the sense as explained in the previous section. The epistemological limitations of the notion of prediction are useful to set the problem in the context of $\mathrm{AI}$ and $\mathrm{SB}$, where the logical consequences of scientific theories are effective artifacts or entities with a specific open-ended behavior.

\section{Prediction in Artificial Intelligence and Synthetic Biology}

Prediction is connected to different fields of AI and cognitive science. There are at least four areas in which prediction is involved: $(a)$ in computability theory, where we want to know if a computer program and a specific input will end or if it will go on forever. This is also known as the halting problem and it is one of the first problems to be proven as undecidable by Turing (see [13]) and Church (see [14]). It is still a programming problem, because we would like to know if a complex program with a certain input will stop and we want to know this with as much accuracy as possible. In other terms, we want to predict the running of the program in the best possible way. The halting problem is a logical obstacle to this requirement for the programs, a hard one as regards prediction; $(b)$ prediction is important for game theory and decision theory, whose aims are, among others, to predict the agent's behavior in an interactive situation or in a choice situation, and where one of the crucial notions is that of expected utility; $(c)$ prediction is significant in the simulative approach in the science of the artificial, and with reference to the synthetic method (see [15]), to build and exploit software and/or hardware models and artifacts with a rational/intelligent/cognitive behavior. The way in which we build the model, or the artifact as a model, includes elements the builder 
thinks the model should have to show a specific behavior, which of course could also be disregarded in the case of a wrong prediction; and $(d)$ prediction is important in understanding future technological developments and their impacts. This is especially true for AI and its aim to achieve a general artificial intelligence or a human-like artificial intelligence with powers that we do not understand yet, and the risks of no control are high.

I claim that $(a),(c)$ and $(d)$ concerning AI are relevant for SB. The problem of prediction of algorithms has a corresponding problem in new genetic editing technologies. The problem of prediction in the simulation of evolutionary techniques of AI, and in AI self-replicating systems and cellular automata, has a corresponding problem in SB emergent phenomena and multicellularity issues. The problem of prediction in relation to artificial superintelligence building has an analogous problem in the general aim of SB complex systems building. These are the three problems of prediction I will address as follows.

\section{The Three Problems of Prediction}

\subsection{Algorithm Prediction in Artificial Intelligence and Synthetic Biology}

In computability theory, decidable problems, unlike the halting problem, are those that are computable in a finite number of steps, but we do not know how many steps and which ones. They are solvable by a Turing machine (TM), according to the Church-Turing thesis-actually not a thesis but a conjecture that was never disproven. The Church-Turing thesis is a model of computation. It is based on the abstraction of computers with an infinite amount of memory. A TM-solvable problem needs a finite, even though indefinite, amount of memory. The algorithm concludes, namely the program stops, but the greater the complexity of the algorithm and its inputs, the harder it is to know when the program stops and its output. From an epistemic standpoint, computation is often beyond the limits of human knowledge.

This problem has been computationally and mathematically addressed since the mid-1960s. From this point of view, this field of study is known as Kolmogorov complexity and was independently started by Andrei Kolmogorov, Ray Solomonoff and Gregory Chaitin (for a historical reconstruction, see [16]). In particular, Solomonoff claimed that it is possible to consider the complexity of a binary string $s$ as the length of the shortest program that, starting from no input, prints out $s$. This definition of complexity helps to find a hierarchy in the complexity of algorithms and assign a probability to every string and to everything that could be coded as binary strings, that is, 
for example, a hypothesis on empirical facts. The less the complexity (the shortest program), the more the probability. (For a discussion of the relationship between algorithmic probability in the context of prediction from an epistemological point of view, see [17]. See also [18] for a discussion of the predictability in deterministic computing systems and the general epistemology of algorithms.) This problem is also connected to the issue of computational irreducibility, the idea that for some physical system it is possible to simulate every step of the evolution of system behavior, but it is not possible to predict an outcome of such evolution without letting the system evolve every step. In other words, there are no shortcuts to predict the behavior of the system. This is called computational irreducibility $[19,20]$ and it is typical of emergent phenomena [21], like biological phenomena and related simulations.

The question of algorithmic complexity is strictly related to computability theory and theoretical computer science. If we consider AI's main assumptions and basic ideas on formal systems within an unpredictable context, that is, a real-world context in which AI systems behave, the notions of prediction and predictability have different senses, concerning the behavior of the AI system, along with the context in which it happens and the limitations of the observer. This was already stated by Turing in the 1950s.

In Turing's most famous paper on machinery intelligence, he claimed that prediction connected to a general issue about machines that are able to think. The first one is in the discussion of Lady Lovelace's objection, according to which a machine "has no pretensions to originate anything. It can do whatever we know how to order it to perform" [22, p. 455]. The point is, if we consider algorithms, that nothing new is in the running of a program, as it will do what it is built for and nothing more. Turing said, however, that "machines take me by surprise with great frequency. This is largely because I do not do sufficient calculation to decide what to expect them to do, or rather because, although I do a calculation, I do it in a hurried, slipshod fashion, taking risks. [...] The view that machines cannot give rise to surprises is due, I believe, to a fallacy to which philosophers and mathematicians are particularly subject. This is the assumption that as soon as a fact is presented to a mind all consequences of that fact spring into the mind simultaneously with it. It is a useful assumption under many circumstances, but one too easily forgets that it is false. A natural consequence of doing so is that one then assumes that there is no virtue in the mere working out of consequences from data and general principles" ([22, pp. 455-456], emphasis added).

One is not aware of all the consequences of a formal system, or of a system presented in a formal way. This is an empirical fact, of course, due to the limits of human cognitive capabilities and bounded 
cognitive systems. Moreover, every program runs on physical hardware that is subject to microphysical perturbations, which undermine the idea of a deterministic universe. This is what Longo [23] calls the "electron effect," which anticipates the "butterfly effect" by Lorentz, referring to Turing's claim in a 1950 paper, "The displacement of a single electron by a billionth of a centimeter at one moment might make the difference between a man being killed by an avalanche a year later, or escaping." Longo sees in it an earlier example of the unpredictability of nonlinear dynamics connected with the sensitivity to initial conditions, pointing out that, actually, the " 1950 article had been read principally in relation to Artificial Intelligence; this did not allow people to grasp [Turing's] insight as a great mathematician who was working on morphogenesis, as a physical dynamic in the continuum" [23]. Turing's work on morphogenesis [24] addressed the breaking of symmetry and homogeneity and the creation of reactiondiffusion systems, which are complex systems for all intents and purposes.

It is true that Turing's 1950 article had been read in relation to AI in a long-lasting debate on the imitation game, the Turing test, and so on. It is also true, however, that AI emphasizes the unpredictable aspects of algorithms especially for two reasons: $(a)$ one of the main aims of $\mathrm{AI}$ is to produce autonomous systems; and $(b)$ AI products interact with an open-ended context. In other terms, processing predictions is hard due to the open-endedness of the real-world context. This is the asymmetry we discussed previously; that is, we can explain every step of a program, but we cannot always predict future steps.

SB has two main streams of research, which usually are expressed in this way: $(a)$ the design and construction of new biological parts, devices and systems; and $(b)$ the redesign of existing natural biological systems for useful purposes. SB also has different subfields: bioinspired and bio-mimetic SB, recombinant DNA applied to metabolic engineering, genome engineering, evolution, and biological building using bio-bricks [25]. Within genome engineering, there is the new and very promising subfield of genome editing, which draws the attention also of all nonexperts because of the potential implications on life, medicine, economy, and so on. Genome editing is the best candidate to a parallelism with the AI algorithmic point of view in SB.

The long-lasting debate on the use of information in biology was developed until the modern techniques of genome editing came about. The use of information terminology to describe molecular biology processes dates to the discovery of DNA [26] and even to the Schrödinger book on life [27]. The core idea is that DNA is genetic information. The processes of gene expression and protein synthesis were soon described with notions such as translation, transcription, code, messenger, editing and others (see, for example, $[28,29])$. In the literature 
of the last 20 years, a different position about DNA as information was argued by many authors (e.g., [30, 31]): that DNA is not information, but information and related terms have a metaphorical or heuristic role in biological research. For better research practices, information should be eliminated from biological explanations. Someone has argued that such a metaphorical use is even dangerous [32]. (Longo underlines that Turing, in the article on morphogenesis [24], claims that "genes are at most the producers of enzymes which are involved in the reaction $[\ldots]$, and it is the speed of this production that contributes to a process that is global, interactive, and based on physical continua, not 'computational,' even less 'programmed'" [32].) On the contrary, some authors defend the role of information theory and algorithmic programming to explain biological phenomena, especially as regards the relationship between disease and the immune system, and in relation to the possibility of reprogramming cells. These software-engineering approaches to systems biology are based on a computational interpretation of cell behavior. Gene therapy is one example of this methodology. The use of algorithmic terminology is an attempt to strictly predict the behavior of the system, and some theoretical concepts of information theory are exploited to control the system exposed to perturbation, such as the cell replication process [33]. The debate is still open. The reprogramming of a cell can be achieved by natural or artificial methods, which is close to some recent developments of SB that use techniques in line with this approach.

Even though there are many criticisms concerning the use of information terms in molecular biology that are considered too vague and misleading, this kind of terminology is present in recent papers about genome editing techniques. My claim is that could have consequences in the SB subfield as regards prediction issues. For example, in a paper on CRISPR-Cas9 [34], the information terminology still remains and is in line with the long tradition of molecular biology. In the introduction, genome engineering is defined as "the process of making targeted modifications to the genome, its contexts (e.g., epigenetic marks), or its outputs (e.g., transcripts); to overcome the challenges of gene-targeting experiments a series of programmable nuclease-based genome editing technologies have been developed in recent years" [emphasis added]. The most famous editing technology is Clustered Regularly Interspaced Short Palindromic Repeats (CRISPR) and its associated systems, especially the Cas9 endonuclease enzyme. Cas9 is the only enzyme within the cas-gene cluster that mediates target DNA cleavage (Figure 1), but the CRISPR-cas coding power has been proven not only in biology but has been used, for example, to encode a digital movie into the genomes of a population of living bacteria [35]. 


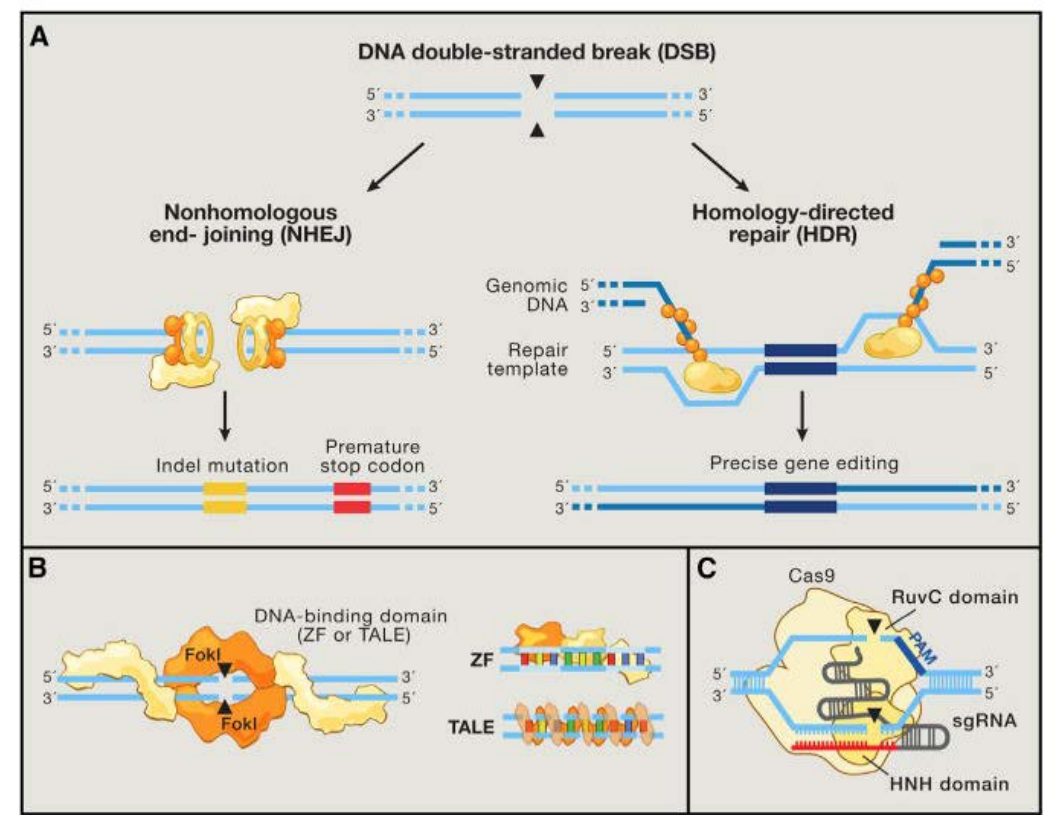

Figure 1. The DNA double-stranded break and in box C, the Cas9 working (from [34]).

CRISPR-cas9 and gene prediction (the process to identify DNA regions connected to genes) techniques are powerful tools. In particular, the CRISPR-cas9 system is usually described in the following manners: it allows DNA sequence cleavage, it can be reprogrammed to target a site by changing the sequence of its crRNA, and it can make programmable transcription factors allowing specific genes to be activated or silenced. It has many different applications: biogenesis, generation of cellular models, generation of transgenic animal models, and so on [34].

However, researchers are aware of some potential problems: "Because genome editing leads to permanent modifications within the genome, the targeting specificity of Cas9 nucleases is of particular concern, especially for clinical applications and gene therapy. A combination of in vitro and in vivo assays has been typically used [...] but systematic analysis has remained challenging due to difficulties in synthesizing large libraries of proteins with varying sequence specificity" [34]. This does not seem to be an in-principle problem, but just a contingency problem, due to the state of the art of technology. "Systematic analysis" will be addressed in the coming years with the development and improvement of techniques and with new experimental achievements. More interesting is a part of the paper's 
abstract, where the reference to the programming domain is explicit: "Analogous to the search function in modern word processors, Cas9 can be guided to specific locations within complex genomes by a short RNA search string. Using this system, DNA sequences within the endogenous genome and their functional outputs are now easily edited or modulated in virtually any organism of choice" [34] (emphasis added). The analogy with word processors is used to stress positively the wide potential use of Cas9. It is this analogy, however, that shows the risks of the Cas9 technique.

One of the acknowledged risks of this technique is the possibility of mosaicism (populations of cells with different genomes in an individual), but this is an "internal," domain-specific risk. If we consider the parallelism with AI and computer algorithms, an external, more general risk is connected to the uncertainty of the consequences of results, that is, to the prediction of the Cas 9 processes. Outcomes are not under control in the long run because there are too many factors involved in gene expression and cell reproduction. In this case, the metaphor of information could be useful to cast some light on the genome editing general perspective. If genome editing becomes a science of programming, or is meant as a science of programming, it can inherit some warning from AI and computation theory related to prediction. Moreover, my claim is that the whole SB, of which genome engineering is a subfield, has the same problem of prediction as AI in the long run because of the following: (a) SB aims at producing autonomous systems; and $(b)$ SB products that interact with an openended context.

\subsection{Prediction and Complex Systems}

A second problem of prediction concerns the complex systems involved in the origins of artificial life. In the Hixon symposium of 1948, von Neumann tackled the problem of self-replicative systems. He tried to establish a logical theory of self-replication by addressing the issue of evolution through errors in replication [36]. After his work, the general theory of cellular automata, that is, spaces in which cells change according to specific rules, was developed in subsequent years. In the logical simulation of self-replication, one may distinguish two different senses of self-replication: (1) of a single entity, a cell; and (2) of systems made by cells replicating themselves. This is a replication and self-replication of complex systems, reproducing themselves at some emergent level. John Conway's Game of Life is a wellknown example of a cellular automaton, and Langton's Ant is one of the most famous applications of cellular automata to artificial life [37]. These are examples of the second case of self-replication and are important as complex systems with emergent unpredictable behavior. 
The study of evolutionary laws and principles that von Neumann began established a bridge between the collective behavior of microentities, from a logical and a mathematical point of view, as well as emergent phenomena in the biological domain. This bridge connects $\mathrm{AI}$ and biology from a bottom-up standpoint and according to the perspective of the emergence of complex system behavior from the interaction of the system's parts. This led to evolutionary computation and evolutionary programming, which are relevant techniques in AI, artificial life and general computer science. In particular, genetic algorithms are the basis of the complex adaptive systems developed since the 1970s [38]. The emergence of new entities in space or time, which is typical of evolution, is an unpredictable process, even though some attempts to go beyond this problem have recently been made [21]. In evolutionary computation, such unpredictability is not only accepted but is also the strength of these kinds of techniques. So where is the problem? The problem lies in the fact that the strength given by unpredictability is due to the system being without control. In an artificial simulation, this is not a problem, but what about in an actual biological synthetic system?

SB has complex systems as outcomes or targets, for example, synthetic multicellular systems $[39,40]$. Evolutionary techniques in SB engineering are useful for in vitro experiments, but in general, SB does not like overwhelmingly emergent phenomena [41], because it is hard to control SB products if their properties, development and behavior are emergent, especially when SB products have to be inserted in the real world. To study emergent entities, behaviors and products of evolutionary processes, AI uses computer simulation and models, especially in the framework of the synthetic method [15]. The test bench of synthetic method outcomes, however, is often (e.g., in robotics) the interaction with the real world. Negative, namely nonadaptive, autonomous entities or behaviors are changed or deleted. In SB systems with emergent properties, the consequences, the behavior and the new emergent entities are as unpredictable as in AI. SB may use in silico and in vitro analysis and models (see, for example, [42]). But what about in vivo SB entities? Is it possible to use them to predict consequences and emergent properties? Is it possible to eliminate or change negative in vivo products, maybe inside living organisms, in the same way as in the case of AI modeling and artifacts? Do or could $\mathrm{AI}$ and SB share the same sort of simulation method and entity building? The answers to these questions are related to the possibility of making predictions and using techniques that are in principle unpredictable as regards their outcomes. Unpredictability is constitutive of these methods, but in the real world of living systems, it may become a problem if there are no means to control the outcomes of unpredictable processes. The possibility of control seems to be a minimal 
requirement in every aspect related to this problem, the unpredictability of emergence, in SB.

\subsection{Prediction and General Artificial Intelligence and Synthetic Biology Aims}

In his 1950 paper, Turing made a second claim concerning prediction about computing machinery and intelligence: "I believe that in about fifty years' time it will be possible, to programme computers [...] to make them play the imitation game so well that an average interrogator will not have more than 70 per cent chance of making the right identification after five minutes of questioning. [... ] I believe that at the end of the century the use of words and general educated opinion will have altered so much that one will be able to speak of machines thinking without expecting to be contradicted. I believe further that no useful purpose is served by concealing these beliefs. The popular view that scientists proceed inexorably from well-established fact to well-established fact, never being influenced by any improved conjecture, is quite mistaken. Provided it is made clear which are proved facts and which are conjectures, no harm can result. Conjectures are of great importance since they suggest useful lines of research" [22, p. 449].

The issue of Al's future is hotly debated today. Turing was one of the first thinkers who dealt with this issue. His predictions have been wrong about passing the test (the imitation game), but rather exact about speaking of machine thinking at the end of the twentieth century. The "importance of the conjectures," underlined by Turing, is significant in the present-day debate on the future of AI. In particular, many authors discuss the possibility of an artificial general intelligence and of a (biological or artificial) superintelligence [43], that is, an intelligence that exceeds human intelligence. Even though the future is mostly unpredictable, this is another relevant problem for prediction in AI, as it is not clear if we already have or we will soon have technological achievements enabling artificial or biological entities that are more intelligent than human beings. The problem is not trivial, as there are at least two cases: (1) a superintelligence we can recognize for its power to do things we want it to do, but are unable to do; and (2) a superintelligence that we will not recognize, as its powers, goals, motivations and methods are far from human understanding. The former case is more predictable than the latter, and the problem is that in the former case it is most likely that we will be in control of the superintelligent entity, whereas we will not in the latter case, because of our not understanding the new superintelligent entity. Prediction is crucial, but it is hard. Therefore, nowadays there are many institutes and centers of research dealing with this problem. 
(For example, the Future of Humanity Institute, the Future of Life Institutes and the Leverhulme Center for the Future of Intelligence, among others.)

SB is part of this scenario as well. For example, in a technological improved scenario, genetic manipulation, selection and engineering, including genome editing techniques, could lead to biological superintelligence through understanding the biological mating patterns behind intelligence, or maybe in some other differently controlled evolutionary ways. Consider, as an example of methodology, implantation in embryos and embryo selection over many generations. This may lead to a weak form of superintelligence, a biological one, which could produce smarter and smarter human beings by accelerating the evolutionary process. Within the framework of "transhumanism" $[25,44]$, the point is that a great number of more and more intelligent humans will be able to produce artificial superintelligences. SB techniques and methods can provide control of transhuman entities [25]. Further, in this way, SB could help future AI by solving part of the problem of prediction if $\mathrm{SB}$ is able to control its processes and outcomes.

From the point of view of AI, evolutionary computation seems to be a good candidate in the attempt to achieve an artificial superintelligence, insofar as human intelligence is a product of evolution, according to Moravec's predictions [45]. But how? By simulating the features of evolutionary process? Or by increasing computation power to exploit the existing evolutionary computational technology in a fuller way? The problem of predicting the outcomes of processes still remains and so the problem controls them. The outcomes of evolutionary computation could help to achieve or avoid similar developments in SB. So the debate and research on artificial superintelligence can be used to gain some insight into current research on AI general and specific systems, as well as in SB. This kind of guiding role of future predictions concerning general complex systems seems to be useful in this third version of the prediction problem. In particular, the regulative role of these ideas-that is, prediction concerning the developments of unprecedented and autonomous complex systems that could have a deep influence on human life and society-may help to define the ethical, legal and political regulation of $\mathrm{AI}$ and $\mathrm{SB}$, a field debated in the present-day political agenda of public and private institutions.

\section{Conclusion}

In this article I have established an analogy between artificial intelligence $(\mathrm{AI})$ and synthetic biology $(\mathrm{SB})$ in relation to the problem of 
prediction, which I have characterized in three different manners concerning the three subfields of AI and SB: algorithms and programming; complex systems and emergence; and epistemological issues of the future of these disciplines. My main claim is that the analogy relies on the aim of $\mathrm{AI}$ and $\mathrm{SB}$ to produce autonomous systems and systems interacting with an unpredictable context, that is, the real (environmental and social) world. My suggestion is that such an analogy is helpful for generating warnings on research methods and targets, for discussing the limits and the possibilities of both AI and SB, for steering the research trends and for understanding what is next in these adjacent fields, at least as regards their general aim of producing autonomous (complex) systems. The knowledge produced in AI and SB is useful if it is shared by researchers of both fields, in both directions, and on the basis of similar theoretical terminology, whenever this sharing is possible.

\section{References}

[1] European Group on Ethics in Science and New Technologies. "Statement on Artificial Intelligence, Robotics and 'Autonomous' Systems.” (Aug 14, 2018)

ec.europa.eu/research/ege/pdf/ege_ai_statement_2018.pdf.

[2] H. Hesse, "Autonomous Systems and Synthetic Biology," in Frontiers of Engineering: Reports on Leading-Edge Engineering from the 2010 Symposium, Washington, DC: The National Academies Press, 2011 pp. 149-158. doi:10.17226/13043.

[3] E. Nagel, The Structure of Science: Problems in the Logic of Scientific Explanation, New York: Harcourt, Brace \& World, 1961.

[4] M. A. Bedau, “Weak Emergence,” Nô̂s, 31(s11), 1997 pp. 375-399. doi:10.1111/0029-4624.31.s11.17.

[5] M. A. Bedau and P. Humphreys (eds.), Emergence: Contemporary Readings in Philosophy and Science, Cambridge, MA: MIT Press, 2008.

[6] F. C. Boogerd, F. J. Bruggeman, R. C. Richardson, A. Stephan and H. V. Westerhoff, "Emergence and Its Place in Nature: A Case Study of Biochemical Networks," Synthese, 145(1), 2005 pp. 131-164. doi:10.1007/s11229-004-4421-9.

[7] D. Noble "A Theory of Biological Relativity: No Privileged Level of Causation," Interface Focus, 2(1), 2012 pp. 55-64. doi:10.1098/rsfs.2011.0067.

[8] C. G. Hempel, Aspects of Scientific Explanation, and Other Essays in the Philosophy of Science, New York: Free Press, 1965. 
[9] D. M. Hausman, Causal Asymmetries, New York: Cambridge University Press, 1998.

[10] J. F. Woodward, Making Things Happen: A Theory of Causal Explanation, New York: Oxford University Press, 2003.

[11] N. Rescher, The Limits of Science, Berkeley: University of California Press, 1984.

[12] N. Rescher, Predicting the Future: An Introduction to the Theory of Forecasting, Albany, NY: State University of New York Press, 1998.

[13] A. M. Turing, "On Computable Numbers, with an Application to the Entscheidungsproblem," Proceedings of the London Mathematical Society, 42, 1936 pp. 230-265. doi:10.1112/plms/s2-42.1.230.

[14] A. Church, "An Unsolvable Problem of Elementary Number Theory," American Journal of Mathematics, 58(2), 1936 pp. 345-363. doi:10.2307/2371045.

[15] R. Cordeschi, "Steps toward the Synthetic Method: Symbolic Information Processing and Self-Organizing Systems in Early Artificial Intelligence Modeling," in The Mechanical Mind in History (P. Husbands, O. Holland and M. Wheeler, eds.), Cambridge, MA: MIT Press, 2008 pp. 219-258. doi:10.7551/mitpress/9780262083775.003.0010.

[16] M. Li and P. M. B. Vitányi, An Introduction to Kolmogorov Complexity and Its Applications, 2nd ed., New York: Springer, 1997.

[17] W. Kirchherr, M. Li and P. Vitányi, "The Miraculous Universal Distribution," The Mathematical Intelligencer, 19(4), 1997 pp. 7-15. doi:10.1007/BF03024407.

[18] H. Zenil, F. Soler-Toscano and J. J. Joosten, "Empirical Encounters with Computational Irreducibility and Unpredictability," Minds and Machines, 22(3), 2012 pp. 149-165. doi:10.1007/s11023-011-9262-y.

[19] S. Wolfram, A New Kind of Science, Champaign, IL: Wolfram Media, Inc., 2002.

[20] H. Zwrin and J.-P Delahaye, "Unpredictability and Computational Irreducibility," in Irreducibility and Computational Equivalence: Emergence, Complexity and Computation, Vol. 2 (H. Zenil, ed.), Berlin, Heidelberg: Springer-Verlag, 2013 pp. 273-295. doi:10.1007/978-3-642-35482-3_19.

[21] H. Zwrin, "Computational Irreducibility and Computational Analogy," Complex Systems, 24(2), 2015 pp. 149-174.

doi:10.25088/ComplexSystems.24.2.149.

[22] A. M. Turing, "Computing Machinery and Intelligence," Mind, 59(236), 1950 pp. 433-460, (reprinted in The Essential Turing: Seminal Writings in Computing, Logic, Philosophy, Artificial Intelligence, and Artificial Life, Plus the Secrets of Enigma (B. J. Copeland, ed.), New York: Oxford University Press, 2004, pp. 441-464). 
[23] G. Longo, "Letter to Turing," Theory, Culture and Society, Posthumanities, Special Issue on "Transversal Posthumanities" (M. Fuller and R. Braidotti, eds.), Jun 7, 2018. doi:10.1177/0263276418769733.

[24] A. M. Turing, “The Chemical Basis of Morphogenesis," Philosophical Transactions of the Royal Society of London, Series B, 237(641), 1952 pp. 37-72. doi:10.1098/rstb.1952.0012.

[25] G. M. Church and E. Regis, Regenesis: How Synthetic Biology Will Reinvent Nature and Ourselves, New York: Basic Books, 2012.

[26] J. D. Watson and F. H. C. Crick, "Genetical Implications of the Structure of Deoxyribonucleic Acid," Nature, 171, 1953 pp. 964-967. doi:10.1038/171964b0.

[27] E. Schrödinger, What Is Life? The Physical Aspect of the Living Cell, Cambridge, UK: Cambridge University Press, 1944.

[28] J. Monod, Le hasard et la nécessité. Essai sur la philosophie naturelle de la biologie moderne, Paris: Seuil, 1970.

[29] D. R. Hofstadter, Gödel, Escher, Bach: An Eternal Golden Braid, New York: Basic Books, 1979.

[30] S. Sarkar, "Information in Genetics and Developmental Biology: Comments on Maynard Smith," Philosophy of Science, 67(2), 2000 pp. 208-213. doi:10.1086/392771.

[31] G. Boniolo, "Biology without Information," History and Philosophy of the Life Sciences, 25(2), 2003 pp. 255-273.

www.jstor.org/stable/23332431.

[32] G. Longo, "The Biological Consequences of the Computational World: Mathematical Reflections on Cancer Biology," Organisms. Journal of Biological Sciences, forthcoming.

[33] H. Zenil, A. Schmidt and J. Tegnér, "Causality, Information, and Biological Computation: An Algorithmic Software Approach to Life, Disease, and the Immune System," From Matter to Life: Information and Causality (S. I. Walker, P. C. W. Davies and G. F. R. Ellis, eds.), New York: Cambridge University Press, 2017 pp. 244-279.

[34] P. D. Hsu, E. S. Lander and F. Zhang, "Development and Applications of CRISPR-Cas9 for Genome Engineering,” Cell, 157(6), 2014 pp. 1262-1278. doi:10.1016/j.cell.2014.05.010.

[35] S. L. Shipman, J. Nivala, J. D. Macklis and G. M. Church, "CRISPRCas Encoding of a Digital Movie into the Genomes of a Population of Living Bacteria," Nature, 547, 2017 pp. 345-349. doi:10.1038/nature23017.

[36] J. von Neumann, "The General and Logical Theory of Automata," in Cerebral Mechanisms in Behavior: The Hixon Symposium (L. A. Jeffress, ed.), New York: Wiley, 1951 pp. 1-41. 
[37] C. G. Langton, "Studying Artificial Life with Cellular Automata," Physica D: Nonlinear Phenomena, 22(1-3), 1986 pp. 120-149. doi:10.1016/0167-2789(86)90237-X.

[38] J. H. Holland, Adaptation in Natural and Artificial Systems: An Introductory Analysis with Applications to Biology, Control, and Artificial Intelligence, 1st MIT Press ed., Cambridge, MA: MIT Press, 1992.

[39] J. S. Markson and M. B. Elowitz, "Synthetic Biology of Multicellular Systems: New Platforms and Applications for Animal Cells and Organisms," ACS Synthetic Biology, 3(12), 2014 pp. 875-876.

doi:10.1021/sb500358y.

[40] M. M. Maharbiz, "Synthetic Multicellularity," Trends in Cell Biology, 22(12), 2012 pp. 617-623. doi:10.1016/j.tcb.2012.09.002.

[41] D. Endy, "Foundations for Engineering Biology," Nature, 438, 2005 pp. 449-453. doi:10.1038/nature04342.

[42] G. Fiore, A. Matyjaszkiewicz, F. Annunziata, C. Grierson, N. J. Savery, L. Marucci and M. di Bernardo, "In-Silico Analysis and Implementation of a Multicellular Feedback Control Strategy in a Synthetic Bacterial Consortium," ACS Synthetic Biology, 6(3), 2017 pp. 507-517. doi:10.1021/acssynbio.6b00220.

[43] N. Bostrom, Superintelligence: Paths, Dangers, Strategies, Oxford, UK: Oxford University Press, 2014.

[44] E. Regis, Great Mambo Chicken and the Transhuman Condition: Science Slightly over the Edge, Reading, MA: Addison-Wesley, 1990.

[45] H. Moravec, Mind Children: The Future of Robot and Human Intelligence, Cambridge, MA: Harvard University Press, 1988. 